\title{
Regulation of mRNA stability through a pentobarbital-responsive element
}

\author{
Bünyamin Akgül a,b,c , Chen-Pei D. Tu ${ }^{\mathrm{a}, \mathrm{b}, *}$ \\ ${ }^{a}$ Department of Biochemistry and Molecular Biology, The Pennsylvania State University, 108 Althouse Lab, University Park, PA 16802, USA \\ ${ }^{\mathrm{b}}$ Institute of Biomedical Sciences, Academia Sinica, Taipei 11529, Taiwan, ROC \\ ${ }^{c}$ Department of Biology, Izmir Institute of Technology, Urla, Izmir 35430, Turkey
}

Received 23 August 2006, and in revised form 22 October 2006

Available online 2 November 2006

\begin{abstract}
Pentobarbital, a general anesthetic and non-genotoxic carcinogen, can induce gene expression by activating transcription. In the Drosophila glutathione $S$-transferase D21 (gstD21) gene, pentobarbital's regulatory influence extends to the level of mRNA turnover. Transcribed from an intronless gene, gstD21 mRNA is intrinsically very labile. But exposure to pentobarbital renders it stabilized beyond what can be attributed to transcriptional activation. We aim here to identify cis-acting element(s) of $g s t \mathrm{D} 21 \mathrm{mRNA}$ as contributors to the molecule's pentobarbital-mediated stabilization. In the context of hsp70 5'UTR and the 3'UTR of act5C, gstD21 mRNA, minus its native UTRs, is stable. Maintaining the same context of heterologous UTRs, we can reconstitute using the full-length gstD21 sequence the inherent instability of $g s t \mathrm{D} 21 \mathrm{mRNA}$ and its stabilization by pentobarbital. Transgenic flies that express these chimeric gst 21 mRNA exhibit decay intermediates lacking 3'UTR, which are not stabilized by PB treatment. The 3'UTR sequence, when inserted downstream from a reporter transcript, stabilizes it 1.6-fold under PB treatment. The analysis of the decay intermediates suggests a polysomeassociated decay pattern. We propose a regulatory model that features a 59-nucleotide pentobarbital-responsive element (PBRE) in the 3'UTR of $g s t \mathrm{D} 21 \mathrm{mRNA}$.

(C) 2006 Elsevier Inc. All rights reserved.
\end{abstract}

Keywords: Pentobarbital; Heat shock; Polysome; miRNA; mRNA decay

Glutathione $S$-transferases (GSTs ${ }^{1}$, EC 2.5.1.18) are multifunctional proteins involved in xenobiotic metabolism, drug biotransformation, and protection against peroxidative damage in organisms ranging from bacteria to humans $[1,2]$. There appear to be twenty-five families among over 100 GST-like proteins, each is as different from the next as are the currently recognized classes of alpha, mu, pi, sigma, theta, and kappa isozymes [3]. Each isozyme recognizes a spectrum of substrates. Isozymes from the same gene family and ultimately the superfamily as a whole, form an effective network in the detoxifica-

\footnotetext{
${ }^{*}$ Corresponding author. Fax: +1 8148637024.

E-mail address: unh@psu.edu (C.-P.D.Tu).

${ }^{1}$ Abbreviations used: GST, glutathione $S$-transferase; PB, pentobarbital; PBREM, PB-responsive enhancer module; PBRE, PB-responsive element; HSPB, heat shock-PB experiment; AS, antisense; ARE, AU-rich element.
}

tion of xenobiotics. GSTs catalyze the conjugation of the glutathione thiolate anion to a variety of hydrophobic molecules with reactive electrophilic centers [4-6]. The more hydrophilic glutathione conjugates are further metabolized into mercapturic acid derivatives for excretion. Some GSTs are also capable of binding a diverse group of lipophilic compounds with high affinity (e.g. ligandins), reducing their intrinsic cytotoxicity and facilitating their intracellular transport.

Pentobarbital (PB), a general anesthetic with non-genotoxic carcinogenic properties, belongs to a group of chemicals that induce gene expression by activating transcription. These agents, which include barbiturates, chloropromazine, DDT, act through the nuclear receptors CAR and RXR at the PB-responsive enhancer module (PBREM) of the responsive genes, and eventually stimulate hepatocyte growth [7-10]. 
We discovered earlier, using the Drosophila glutathione $S$-transferase D21 (gst D21) mRNA as a reporter, that PB also can regulate gene expression at the level of mRNA turnover and may affect the choice of its polyadenylation sites $[11,12]$. This gene is actively transcribed, but steadystate levels of its mRNA are, under normal conditions, very low, sometimes barely detectable. Remarkably, however, the $g s t \mathrm{D} 21$ mRNA can be induced $\sim 18$-fold by exposure to PB. With PB treatment, we observed a 2 -fold increase in transcription rate which, though significant, cannot be solely responsible for an overall $\sim 18$-fold increase [11]. We thus suspect that gst $\mathrm{D} 21 \mathrm{mRNA}$, a molecule too labile under normal conditions, must somehow be stabilized in the presence of PB.

We observed that the coding region alone of gst $\mathrm{D} 21$ mRNA is quite stable under control, heat shock, and/or PB treatment conditions. But, in the experimental context of heterologous UTRs, the sequence exhibits no response to $\mathrm{PB}$. In the same context, if we replace the coding region with the complete gstD21(L) mRNA sequence we can reconstitute the destabilization and PB-responsiveness of the corresponding mRNA. The 3'UTR sequence, when placed immediately downstream from a reporter transcript, also renders this transcript relatively stable in the presence of $\mathrm{PB}$. With these results, and additional indication of a major putative decay intermediate that terminates immediately after the $3^{\prime}$ end of the protein coding sequence, we have strong evidence that the $g s t \mathrm{D} 21$ mRNA contains in its
3'UTR a cis-acting, PB-responsive element (PBRE), which is, to a large extent, responsible for PB-mediated stabilization of the gst $\mathrm{D} 21 \mathrm{mRNA}$.

\section{Materials and methods}

\section{Materials}

Bacteriological media, chemicals, and enzymes were described previously [12]. Tobacco acid pyrophosphatase was a product of Epicentre Technologies (Madison, WI). RPA III kits and SUPERase-In ${ }^{\mathrm{TM}}$ RNase inhibitor were purchased from Ambion (Austin, TX). pGEM-T Easy vector was purchased from Promega (Madison, WI). The plasmid vector pCaSpeR-hs-act for Drosophila transformation was obtained from Thummel of the University of Utah [13].

\section{Preparation of transgenic constructs and microinjection}

Transgenes D21L-UTR, D21L, and D21-170-D1, constructed in the same heterologous context, were described previously [12,14]. Briefly, the transgene D21L contains the full-length $g s t \mathrm{D} 21 \mathrm{cDNA}$ cloned in between the 5'UTR of hsp70 and actin5C 3'UTR, whereas D21L-UTR contains only the coding region. D21-170-D1 is a chimeric construct where the Nterminal 170 aa residues of $g s t \mathrm{D} 1$ were replaced with those of $g s t \mathrm{D} 21$. In order to construct D21-170-D1+D21UTR (Fig. 1), the 3'UTR of gstD21 was PCR amplified using the primer sets D21-1 (5'-CGGGATCCTCGGT ATCACAACTATTTATTG-3') and D21-81 (5'-CGGGATCCACTCT GAACAACACATAATAATAA-3') and the EcoRI-digested fragment was cloned into D21-170-D1. The sequence of the insert in the vector was confirmed prior to microinjection. Injection of embryos was performed according to previously described procedures $[12,15]$. Three independent lines for each construct were maintained. We designed the transgenes such

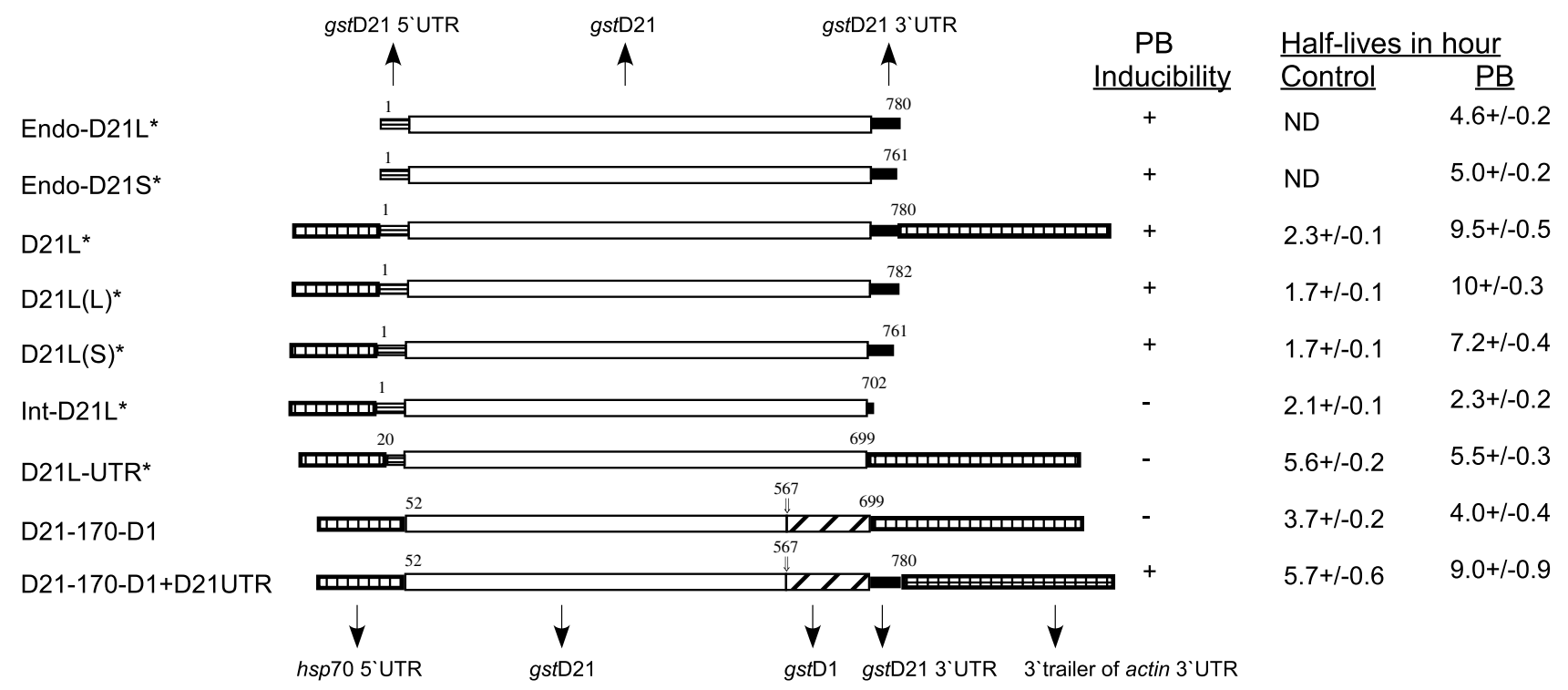

Fig. 1. The half-lives of $g s t \mathrm{D} 21$ deletion lines and a reporter transcript with (D21-170-D1+D21UTR) or without (D21-170-D1) the gstD21 3'UTR. The half-lives (in hours) with SD were calculated from the slopes (slope $=-k / 2.303$ where $k$ is the first order decay rate constant) of semi-log plots of mRNA decay patterns (i.e. $\log [\%$ RNA] vs time, not shown). The half-lives $(t 1 / 2)$ were then calculated by the relationship of $t 1 / 2=0.693 / k$. We used data points from 2 to $10 \mathrm{~h}$ of the decay patterns for the calculation of half-lives to minimize contributions of continued induction by residual PB inside the flies after termination of induction. Each data point was the average of 3-4 experiments (see Fig. S1). Nucleotides 1-780 represent the complete gstD21(L) mRNA sequence (5'UTR 1-52; 3'UTR 700-762 and 700-780 for the S and L forms, respectively). A plus (+) sign indicated PB-mediated stabilization of a given D21 transcript. Endo-D21S and Endo-D21L represented the short and long forms of endogenous gstD21 mRNAs, respectively. D21L, the full-length transgene, which also generates the short $[\mathrm{D} 21 \mathrm{~L}(\mathrm{~S})]$ and long $[\mathrm{D} 21 \mathrm{~L}(\mathrm{~L})]$ forms in addition to decay intermediates $(\mathrm{Int}-\mathrm{D} 21 \mathrm{~L})$. The transgenic $\mathrm{D} 21 \mathrm{~L}(\mathrm{~L})$ has two extra Gs from the pCaSpeR-hs-act vector prior to its polyadenylation (see text). D21L-UTR is similar to D21L except it lacts the 3'UTR of gstD21. D21-170-D1, which lacks the native $3^{\prime} \mathrm{UTR}$, is a chimeric trangene with amino acids 170-215 replaced with that of gstD1. The insertion of the native 3'UTR generates D21-170-D1+D21UTR. *, Ref. [12]. 
that all transcripts would possess the $5^{\prime} \mathrm{UTR}$ of $h s p 70$ and the actin5C 3'UTR of the pCaSpeR-hs-act vector [13].

\section{Pentobarbital and heat shock treatments}

Adult flies (2-3-day-old) were distributed into clean milk bottles in approximately equal numbers for $5 \mathrm{~h}$ starvation at room temperature (21$23^{\circ} \mathrm{C}$ ). Control flies subsequently received a blotting paper strip $(3 \times 10 \mathrm{~cm})$ saturated with a solution of $5 \%$ sucrose; PB-treated flies received a strip soaked in $5 \%$ sucrose plus $200 \mathrm{mg} / \mathrm{mL}$ PB. Strips were placed in the fly bottles for $2 \mathrm{~h}$ at room temperature. Heat shock treatment involved a $1 \mathrm{~h}$ incubation of flies at $35^{\circ} \mathrm{C}$ in clean bottles containing paper strips with $5 \%$ sucrose. Flies in the combined heat shock-PB experiment (HSPB) were subject to PB treatment at room temperature for $1 \mathrm{~h}$ and then at $35^{\circ} \mathrm{C}$ for the second hour. These conditions (HSPB) would jointly induce both the endogenous and transgenic $g_{s t} \mathrm{D} 21 \mathrm{mRNAs}$ [12].

Following treatment of the flies, we set out to determine the time course of decay of transgenic and endogenous $g s t \mathrm{D} 21 \mathrm{mRNAs}$. Flies were allowed to recover at room temperature in milk bottles containing a paper strip soaked in $5 \%$ sucrose for varying durations: $0,0.25,0.5,1,1.5,2,3,4$, $6,8,10$, and $12 \mathrm{~h}$. The flies were subsequently snap-frozen in liquid nitrogen and stored at $-70{ }^{\circ} \mathrm{C}$ until use.

\section{RNA isolation and analysis}

RNA was isolated according to the $\mathrm{CsCl}$ gradient centrifugation method [16]. Purified RNAs had a $A_{260} / A_{280}$ ratio of $\geqslant 1.8$. Templates used to prepare radiolabeled antisense (AS) riboprobes, pSP64(A).D21AS, and pSP64(A).RP-49AS, were described previously [12]. To construct a riboprobe for RPA analyses of D21-170-D1+D21UTR transgenic transcripts, the sequence flanking the $100 \mathrm{bp}$ of the $h s p 70$ 5'UTR, D21-170-D1, gst $\mathrm{D} 213^{\prime} \mathrm{UTR}$, and $100 \mathrm{bp}$ from the act5C 3' UTR trailer was PCRamplified from pCaSpeR-hs-act-D21-170-D1 [14] using the primer sets pC-Actin-5 (5'-CCACATCTGCTGGAAGGTGGACAGCG-3') and pChsp70-partial (5'-AATTAAAAGTAACCAGCAACCAAG-3'). The resulting PCR fragment was cloned into pGEM-T Easy vector (Promega) and sequenced for verification. RPA analyses were carried out using $40 \mu \mathrm{g}$ of total RNA and in vitro-transcribed, gel-purified radiolabeled riboprobes according to instructions from Ambion. The radioactivity in each protected fragment was determined by a PhosphorImager (Molecular Dynamics, CA) with the ImageQuant software package. The intensity of each band was normalized against that of the RP-49 mRNA.

To determine the $3^{\prime}$ ends of the major decay intermediates at the nucleotide level, circular RT-PCR was carried out. Decapping, RNA ligation, reverse transcription and PCR were performed as described previously [17] with the use of the primer set D21-cPDI-RT-3' (5'-GCCTGCAGC CTTGGCCACCATGATGACCG-3') and D21-PDI-5' (5'-GGGAGCT CGGGCCAGGAGTATGTGGCTGG-3'). The expected size of the PCR product was $540 \mathrm{bp}$. We randomly selected 100 clones for sequencing.

\section{Analysis of polysome profile}

Polysomes were isolated according to a previously published procedure with minor modifications [18]. Briefly, $0.5 \mathrm{~g}$ flies were homogenized in $3.5 \mathrm{~mL}$ of lysis buffer $(100 \mathrm{mM} \mathrm{NaCl}, 10 \mathrm{mM} \mathrm{MgCl}, 30 \mathrm{mM}$ Tris- $\mathrm{HCl}$ (pH 7.5), $0.1 \%$ Triton $\mathrm{X}-100,100 \mu \mathrm{g} / \mathrm{mL}$ cycloheximide and $30 \mathrm{U} / \mathrm{mL}$ SUPERase- $\mathrm{In}^{\mathrm{TM}}$ RNase inhibitor (Ambion)). In a negative contol experiment, $30 \mathrm{mM}$ EDTA was added to the lysis buffer to distinguish polysomes from other potential large mRNP complexes. After 5 min of incubation on ice, the homogenates were centrifuged for $8 \mathrm{~min}$ at $12,000 \mathrm{~g}$ at $4{ }^{\circ} \mathrm{C}$. The resulting solution $(1.5 \mathrm{~mL})$ was sedimented in a $5-70 \%(\mathrm{w} / \mathrm{v})$ sucrose gradient by centrifugation for $200 \mathrm{~min}$ at $27,000 \mathrm{rpm}$ at $4{ }^{\circ} \mathrm{C}$ in a Beckman SW28 rotor. Twelve fractions were collected from the top of the gradient using an ISCO Tris pump while monitoring $A_{254}$ with an ISCO UA-6 monitor. Each fraction was extracted by RNase-free phenol/chloroform/ IAA (Ambion) three times. The solution was made to contain $0.5 \%$ SDS prior to the 2 nd extraction. One-third of the total RNA extracted $(\sim 40$
$50 \mu \mathrm{g}$ ) was analyzed for $g s t \mathrm{D} 21$ and related mRNA intermediates by RPA assays according to Ambion's instructions.

\section{Results}

\section{Identification of a PB-responsive cis-acting element in the gstD21 $1 \mathrm{RNA}$}

Most cis-acting elements known to influence mRNA stability lie in the molecule's 5' or 3'UTR [19-23]. Since half-life is the critical measure of mRNA stability, we determined the decay rates of chimeric D21 mRNAs in transgenic flies after transcriptional pulsing by heat shock. With the Drosophila model, short-term activation of the $h s p 70$ promoter by heat shock requires neither transcription inhibition nor in vivo radiolabeling of RNA. To quantify the effect of the UTRs on gstD21 mRNA stability, we calculated changes in the halflives of the HS-induced chimeric D21 mRNAs, both in the presence and absence of $\mathrm{PB}$. We have in fact reported previously that (1) both the long (L) and the short (S) forms of the endogenous gstD21 mRNAs are equally inducible by PB; (2) abrogation of the $3^{\prime} \mathrm{UTR}$ (D21L-UTR) renders it unresponsive to PB treatment, and (3) the PB-induced stabilization could be reconstituted by simply re-inserting the $3^{\prime} \mathrm{UTR}$ (Fig. 1 and Ref. [12]). These observations clearly suggest that the C-terminal 19-nt sequence-the difference between the L and $\mathrm{S}$ forms - and the coding region does not play a role in the PB-mediated stabilization of the gstD21 mRNAs.

To further demonstrate that stabilization is independent of the coding region, we tested the PB-responsiveness of, in addition to D21L-UTR, a series of D21 transgenes without the native UTRs. These transgenes contained a 7-nucleotide prefix, CCCCAAC, ahead of the ATG initiation codon in the 5'UTR (data not shown). In the same context of heterologous UTRs as D21L and D21L-UTR chimeras, the stability of the D21 coding sequence appeared not to be influenced, as expected, by the addition of a 24-nucleotide FLAG sequence at the $\mathrm{C}$-terminus, by mutations in the codons for glycines 8 and $9(\mathrm{G} 8,9 \mathrm{~S})$, or by an extra valine codon at the $\mathrm{N}$-terminus (data not shown). Thus, the native UTRs of D21 mRNA, which were absent from these unresponsive transgenes, must be responsible for the entire molecule's instability under normal conditions. We thus have strong evidence that certain cis-acting element(s) that affect PB-mediated gstD21 mRNA stabilization, which we will call PBRE, pentobarbital-responsive element, reside(s) in the UTRs of the full-length molecule. Since the transgene D21L-UTR, which encodes a stable chimeric D21 mRNA, also contains 32 nucleotides of the native 5'UTR (Fig. 1), this PBRE most likely resides in the 69-nt sequence of the $3^{\prime} \mathrm{UTR}$ of $g s t \mathrm{D} 21$ (L) mRNA and/or in the first 20 nucleotides of the $5^{\prime}$ UTR.

\section{3'UTR of gstD21 mRNA confers PB-mediated stabilization to a reporter $m R N A$ sequence}

Chimeric mRNAs are composites of sequence containing destabilizing and stabilizer elements. They have long 
served as tools in identifying cis-acting elements responsible for mRNA turnover in yeast and mammalian cells [24-29]. We linked the complete $3^{\prime}$ UTR of D21 mRNA downstream of D21-170-D1 [14], a reporter transcript, to test if the 3'UTR would confer PB-mediated stabilization to the reporter D21-170-D1 transcript. Flies carrying D21-170-D1 transgenes with (D21-170-D1+D21UTR) or without (D21170-D1) the native $3^{\prime}$ UTR sequence were heat shocked in the presence and absence of PB to compare the PB-responsiveness of these two transcripts. Our results in Fig. 1 and S1 demonstrated that the half-lives of the reporter D21170 -D 1 transcripts remain nearly the same $(3.7 \pm 0.2 \mathrm{~h}$ vs $4.0 \pm 0.4 \mathrm{~h}$ ) irrespective of PB treatment, whereas the same transcript with the D21 3'UTR becomes PB-responsive $(5.7 \pm 0.6 \mathrm{~h}$ vs $9.0 \pm 0.9 \mathrm{~h})$, resulting in a 1.6 -fold increase in stability after PB treatment. This result supports the notion that the PBRE resides in the 3'UTR of $g s t \mathrm{D} 21 \mathrm{mRNA}$.
Relatively stable putative decay intermediate $(s)$ are devoid of the 3'UTR sequences

We documented previously that the transgenic line D21L contained prominent putative decay intermediates, Int-D21L (Ref. [12], Figs. 1 and 2B, arrows). These major species, which stand out among a background of a slight smear, are smaller than the 658-nucleotide riboprobe-protected chimeric D21L mRNA (Fig. 2C). It is quite intriguing that we have detected such relatively stable decay intermediates. To ascertain that they were not artifacts, we PCR-amplified the transgene from the D21L flies and confirmed the sequence as even a single nucleotide change could have given rise to an artifact of this type in RPA assays. The sequence of the template DNA used for preparation of riboprobes was also confirmed. Additionally, when we induced the endogenous gstD21 mRNAs with PB
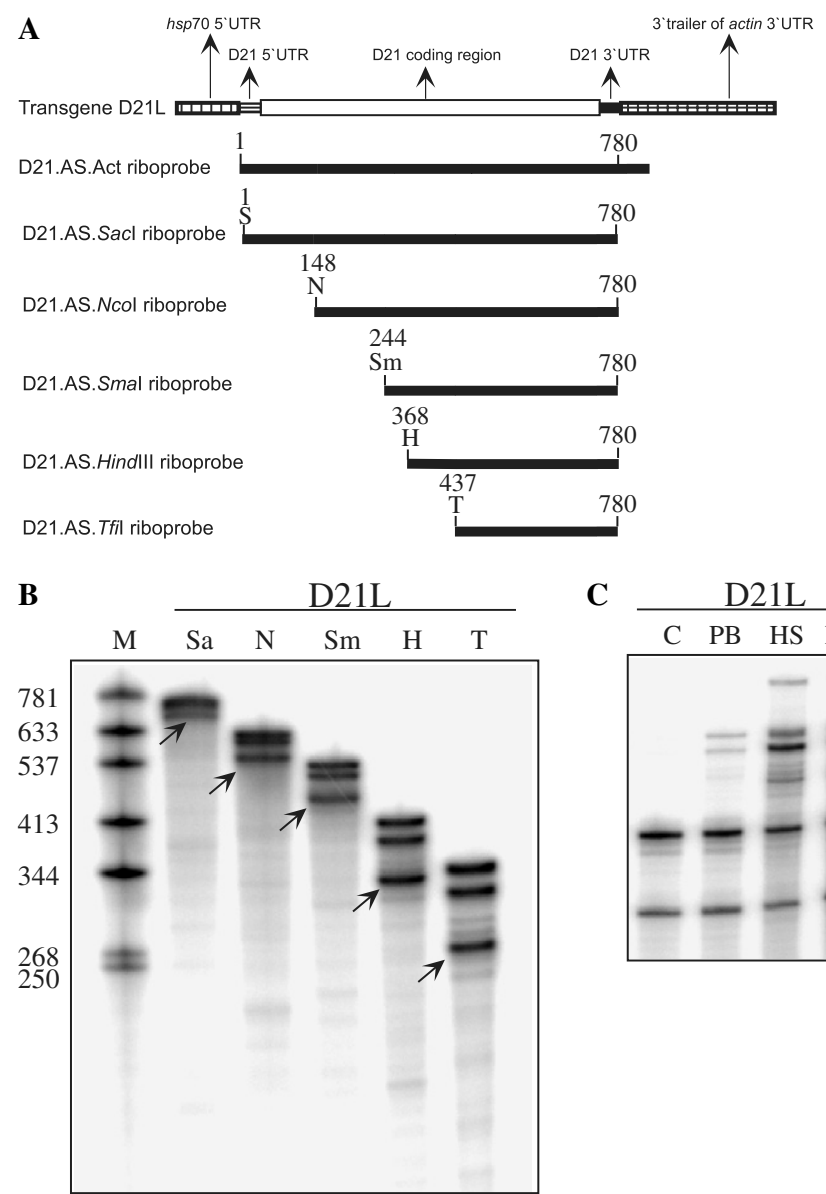

C

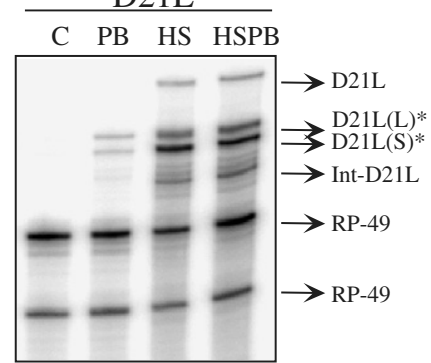

Fig. 2. Mapping the decay intermediates (Int-D21L). (A) A diagram of chimeric D21L mRNA and the riboprobes. A nested set of riboprobes were prepared from $S a c \mathrm{I}(\mathrm{S}), N c o \mathrm{I}(\mathrm{N}), S m a \mathrm{I}(\mathrm{Sm})$, HindIII (H) or TfiI (T) digested D21.AS DNAs. D21.AS.Act riboprobe contains 100 bp sequence from Act5C trailer, which allows to distinguish the endogenous gst $\mathrm{D} 21 \mathrm{mRNA}$ from the chimeric one. Nucleotides $1-780$ represent the complete $g_{s t} \mathrm{D} 21(\mathrm{~L}) \mathrm{mRNA}$ sequence. (B) RPA patterns of Int-D21L using various riboprobes in (A). Total RNAs were isolated from combined heat shock and PB-treated flies (HSPB). Arrows marked positions of Int-D21L protected by each of the riboprobes. The size of each band in the marker lane (M) is indicated to the left of the panel in nucleotides. The calculated size of each protected band from Int-D21L is 690 (SacI probe), 555 (NcoI), 453 (SmaI), 333 (HindIII), and 260 (TfiI) nucleotides, respectively. (C) RPA patterns of Int-D21L. RPA was performed as in (B) except that D21.AS.Act riboprobe was used to distinguish the endogenous and chimeric $g s t \mathrm{D} 21$ mRNAs from each other. D21L(L) and D21L(S) transcripts, which are labeled with an asterisk $(*)$, are a mixture of both endogenous and chimeric gstD21 mRNAs (see text). C, control; PB, pentobarbital; HS, heat shock; HSPB; combined heat shock and pentobarbital treatment. Endogenous gstD21 mRNAs are induced by pentobarbital, whereas transgenic D21L mRNAs are induced only by heat shock treatment. Joint treatment (HSPB) induces both the endogenous and transgenic gst 211 mRNAs. 
treatment (Fig. 2C, PB), the riboprobe did not detect similar decay intermediates, verifying the sequence of the riboprobe. In fact, a closer analysis of the transgenic mRNAs (Fig. 2C, HS and HSPB) reveals the presence of a smear, indicative of a series of decay products. The relatively stable chimeric D21L-UTR mRNA, on the other hand, does not generate any detectable decay intermediates [12]. Under PB influence the decay intermediates from the $\mathrm{D} 21 \mathrm{~L}$ transgene (Int-D21L) are not stabilized relative to the chimeric D21L mRNA (Fig. 1, Int-D21L). This suggests that some segment of the D21 mRNAs remains relatively stable and becomes unresponsive to PB treatment once the PBRE has been removed.

To map the 3' ends of Int-D21L, we used a nested set of riboprobes from D21.AS (Fig. 2B). We linearized the template D21.AS DNA with $S a c \mathrm{I}, N c o \mathrm{I}, S m a \mathrm{I}$, HindIII, or TfiI, to yield riboprobes capable of protecting, respectively, 780, 632, 536, 412, and 343 nucleotides of the gstD21(L) sequence. RPA results showed that the D21.AS.SacI riboprobe-protected a decay intermediate (Int-D21L) of $690 \pm 12$ nucleotides. Fragments protected by other riboprobes below each doublet from the $g_{s t} \mathrm{D} 21(\mathrm{~L})$ and $g_{s t} \mathrm{D} 21(\mathrm{~S})$ mRNAs are marked by arrows. Based on the sizes of the protected fragments, the $3^{\prime}$ end of Int-D21L must lie at or near the translational stop codon (nucleotides $690 \pm 12$, based on the cDNA sequence). Therefore, Int-D21L contains the rest of the D21 coding sequence, up to the detection limit of the $S a c$ I riboprobe (which does not reach any $h s p 70$ sequence in the $5^{\prime} \mathrm{UTR}$ ).

We established in our previous studies that the chimeric D21 transcripts are induced solely by heat shock treatment, whereas the endogenous gst $\mathrm{D} 21$ transcripts are only induced by PB treatment. The D21L line appeared to express, in the absence of $\mathrm{PB}$ treatment, what appeared to be endogenous gst 21 mRNAs (Fig. 2C, $\mathrm{D} 21 \mathrm{~L}(\mathrm{~L})$, and $\mathrm{D} 21 \mathrm{~L}(\mathrm{~S}))$. This unexpected stabilization of the endogenous gst D21 mRNAs by heat shock treatment alone and their shorter half-lives, as compared to the D21L-UTR RNA [12], led us to examine their 3' end(s) at the nucleotide level. During the characterization of the putative decay intermediates (see below), Int-D21L, we identified full-length clones with poly(A) residues from D21L transgenes that contained two extra Gs following the endogenous $3^{\prime}$ end cleavage site. The analysis of the pCaSpeR-hs-act transformation vector and the genomic gst $\mathrm{D} 21$ sequence revealed that these two $\mathrm{G}$ residues are present only in the vector sequence immediately following the endogenous $3^{\prime}$ end cleavage site. Thus, we concluded that at least some chimeric mRNAs (the $5^{\prime}$ UTR of $h s p 70+$ the full-length $g s t \mathrm{D} 21 \mathrm{cDNA}$ ) from the transgene were processed before the actin $5 \mathrm{C}$ trailer, despite the absence of the cognate D21 downstream element for polyadenylation [30]. These $3^{\prime}$ end processed transgenic D21 mRNAs, which contain the native 3'UTR of gst $\mathrm{D} 21(\mathrm{~L})$ mRNA, are also responsive to $\mathrm{PB}$ treatment, exhibiting a 5-fold increase in half-life upon PB treatment (Ref. [12], Fig. 1, D21L(L), and D21L(S)). This result supports the proposition that a PBRE is present within the native $3^{\prime} \mathrm{UTR}$ of $g s t \mathrm{D} 21 \mathrm{mRNAs}$.

cRT-PCR was performed to identify the $3^{\prime}$ ends of Int$\mathrm{D} 21 \mathrm{~L}$ at the nucleotide level. Self-ligation of RNA molecules followed by RT-PCR with the use of a pair of genespecific primers allowed us to simultaneously examine the intactness of the $5^{\prime}$ and $3^{\prime}$ ends. The sequencing results showed that the majority $(>95 \%)$ of the Int-D21L transcripts had an intact $5^{\prime}$ end (data not shown). The $3^{\prime}$ end of the major Int-D21L species was at position 702 of the gstD21 mRNA (Fig. 3A), which is 3 nucleotides downstream from the translational stop codon. The rest of the $3^{\prime}$ ends are scattered throughout the $3^{\prime} \mathrm{UTR}$, suggesting a decay pattern that may involve $3^{\prime} \rightarrow 5^{\prime}$ degradation (Fig. 3A), although an initial independent endonucleolytic cleavage(s) cannot be ruled out.

Since an 18-fold increase in the amount of $g s t \mathrm{D} 21$ mRNA does not result in any increase in the protein level [11], we hypothesized that mRNA stability could be linked to translation. Polysome profile analysis was carried out to assess the degree to which the gst $\mathrm{D} 21 \mathrm{mRNAs}$ is associated with polysomes. We found that the Int-D21L, as well as HS- and PB-induced gstD21 mRNAs, is associated with polysomes and clearly consists of at least two major species separated by 20-25nt (Fig 3B, Int-D21L, thick arrow). Replacement, in the lysis buffer, of $5 \mathrm{mM} \mathrm{MgCl}_{2}$ with $30 \mathrm{mM}$ EDTA shifted the Int-D21L towards the monosomes (data not shown), supporting the notion of its polysome association. It is interesting to note that the larger of the two major Int-D21Ls is associated with polysomes, whereas the smaller one is associated with monosomes. This observation is consistent with the cRT-PCR analysis (Fig. 3A), which showed two clusters of $3^{\prime}$ end sequences, terminating at positions 702 and 735. Together, these results suggest a polysome-associated decay mechanism that involves $3^{\prime} \rightarrow 5^{\prime}$ degradation.

\section{Discussion}

We have evidence for a cis-acting PBRE in the native $3^{\prime} \mathrm{UTR}$ of the gstD21 mRNA, which is essential for PBmediated stabilization. Supporting evidence comes from the following observations: (1) the relative stability of the decay intermediate Int-D21L, which lacks the 3'UTR, does not change with $\mathrm{PB}$ treatment, consistent with the absence of a PBRE in the Int-D21L; (2) the D21L transcript is 2.4fold less stable under control conditions compared to the D21L-UTR transcript, suggesting the presence of a destabilizing element in the native $3^{\prime}$ UTR; (3) the decay intermediate Int-D21L is slightly more stable than the two chimeric D21 mRNAs [D21L(L) and D21L(S)], a result consistent with the destabilizing effect of the $3^{\prime}$ UTR under control conditions; (4) the addition of the $3^{\prime}$ UTR sequence to a PBunresponsive reporter RNA confers PB-responsiveness, confirming a regulatory role of the $3^{\prime}$ UTR in PB-mediated RNA stabilization. Although the $3^{\prime}$ UTR contains a cis-acting PBRE, we cannot address its mechanism of action at 

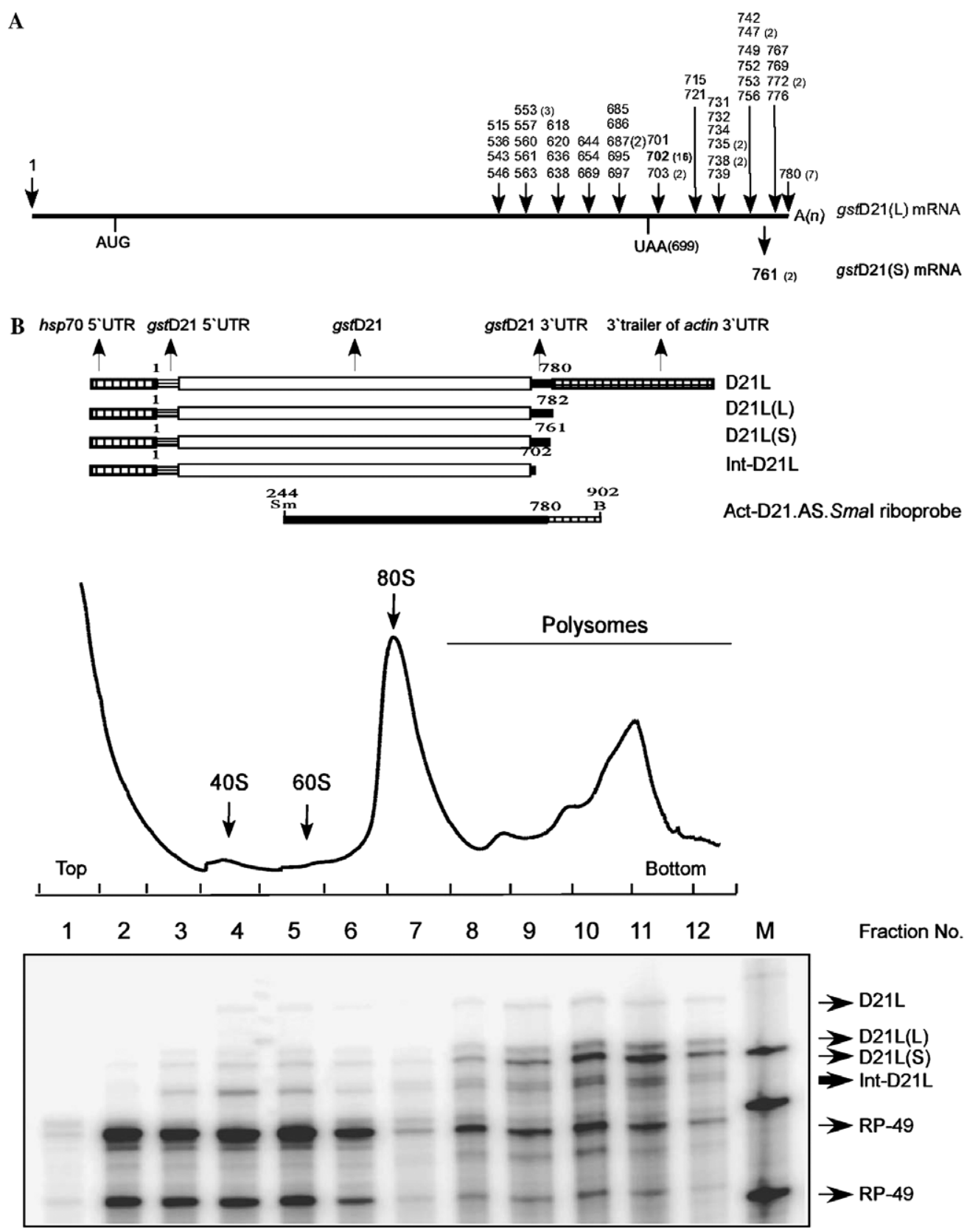

Fig. 3. Polysome-associated decay and identification of two hot spots in the 3'UTR of D21(L) for RNA decay. (A) Total RNA was isolated from HStreated D21L flies. RNA ligation, reverse transcription, and PCR amplification were carried out according to a published procedure [17]. The frequency of each clone is indicated in a parenthesis if detected more than once. Out of 100 clones sequenced, 26 of them were non-informative. (B) Polysome profile analysis of D21 transcripts in the transgenic line D21L. Flies were heat shocked for $1 \mathrm{~h}$ at $35^{\circ} \mathrm{C}$ and polysomal RNA was extracted as explained in "Materials and methods". The top and the bottom of the gradient tube are indicated on top of the RPA gel. Based on the $A_{254}$ profile, fractions 8-12 represent the polysomal fractions. RPA analysis was performed using one-third of the polysomal RNA from each fraction and the Act-D21.AS.SmaI riboprobe. The thick arrow marked the Int-D21 L species. M, markers, 750, 500, 400, and $300 \mathrm{nt}$ (top to bottom).

present. Identification of putative trans-acting factors would be a pre-requisite to substantiate any regulatory model.

Our results indicate that the native 3'UTR is both necessary and sufficient for PB-mediated RNA stabilization. What might be the identity of this PBRE in gstD21 mRNAs? The inducibility by PB of the two forms of the endogenous $g s t \mathrm{D} 21$ mRNAs is about the same. This suggests that the $19 \mathrm{nt}$ at the very $3^{\prime}$ end of the $3^{\prime}$ UTR are not essential for the PBRE function (Fig. 1). Moreover, the lack of PB-responsiveness of the Int-D21 excludes the involvement of the $5^{\prime}$ UTR unless interaction between the $5^{\prime}$ and $3^{\prime}$ UTR is essential. Taken altogether, the comparison of the PB-responsiveness of various D21 transgenes suggests that the PBRE reside in a 59-nt sequence between 702 and 761 (Fig. 1).

Many mRNAs are known to contain destabilizing element(s) in their 3'UTRs [25,31]. One of the best 
characterized examples is the AU-rich element (ARE) found in the 3'UTR of many short-lived, highly inducible mRNAs, such as $c$-fos, $c-m y c, n u r 77$, jun B, IL-3 and GMCSF [23,32]. AREs, which range in size from 50 to 150 nucleotides, encompass a variety of functionally and structurally distinct sequence motifs, such as AUUUA pentamers, UUAUUUA(U/A)(U/A) nonamers, U stretches and/or a U-rich element [25,33]. ARE-binding proteins TTP and BRF1 promote degradation [34,35], whereas HuR increases stability of ARE-containing RNAs [36].

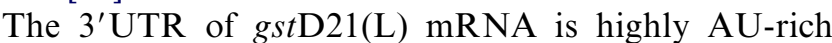
with an AU composition of $77 \%$ and a $U$ content of $43 \%$. The 59-nt-PBRE-containing region, which is $83 \%$ AUrich, contains two scattered AUUUA motifs in a U-rich region, characteristic of Class I AREs [24]. It also has a consensus sequence for the Drosophila ELAV protein RBP9, UUUAUUU [37]. The presence of an ARE, even in a highly AU-rich region ( $>65 \%$ ), while often a good indicator, does not necessarily confer instability [25]. Further studies will determine if the putative class I ARE in the 3'UTR of gst $^{\prime} \mathrm{D} 21 \mathrm{mRNA}$ is in fact responsible for mRNA stability/instability.

Recently, microRNAs, short non-coding singlestranded RNA species found in numerous organisms, have emerged as a new means of gene regulation that can control both translation and mRNA stability depending on the extent of complementarity between the miRNA and its target mRNA $[38,39]$. MiRNA-directed degradation of target RNAs appears to involve an endonucleolytic cleavage at the site of complementarity [40,41]. In HeLa cells, it was shown that AU-rich elements in the $3^{\prime}$ UTR of tumor necrosis factor- $\alpha$ are a target for miR 16 , which contains an UAAAUAUU sequence complementary to the ARE in the target 3'UTR [42]. Interestingly, there is a putative Drosophila miR-5-binding site in the

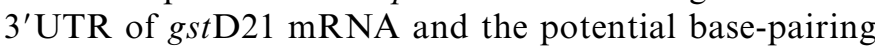
at the $3^{\prime}$ end of the miRNA coincides with the $3^{\prime}$ end of the putative decay intermediate, Int-D21L. It remains to be seen whether any miRNAs are involved in drug-induced mRNA stability.

Several pieces of evidence provided insight into the decay pathway of $g s t \mathrm{D} 21 \mathrm{mRNA}$. The presence of decay intermediates (Fig. 2, Int-D21L), for example, whose $5^{\prime}$ end sequences were intact, and whose $3^{\prime}$ ends mapped immediately after the translational stop codon (Fig. 3A), suggests a possible decay pathway that involves $3^{\prime} \rightarrow 5^{\prime}$ degradation. The abundance of cRT-PCR clones centered around 702 and 735 (Fig. 3A) points to at least two relatively stable decay intermediates, whose $3^{\prime}$ ends, interestingly, are both located within the $3^{\prime}$ UTR of the $g s t \mathrm{D} 21$ sequence. These intermediates were bound to the polysomes. There was a tendency for the smaller one of the two major intermediates to be associated with the monoribosome fraction (Fig. 3B). The mechanistic implications, if any, of this observation remains to be investigated.

\section{Acknowledgments}

We thank Y.-T. Chen and the Institute of Biomedical Sciences for generous support while we were on leave at the Institute of Biomedical Sciences, Academia Sinica between 2003 and 2004. We also thank Henry Y. Sun and C.-Y. Tang of the Institute of Molecular Biology, Academia Sinica for the microinjection of the D21-170D1+D21UTR construct and for fly medium. This work was supported by a grant from the National Institute of Environmental Health Sciences (ES 02678).

\section{Appendix A. Supplementary data}

Supplementary data associated with this article can be found, in the online version, at doi:10.1016/j.abb.2006. 10.026 .

\section{References}

[1] H. Sies, L. Packer, Methods Enzymol. 401 (2005).

[2] J.D. Hayes, J.U. Flanagan, I.R. Jowsey, Annu. Rev. Pharmacol. Toxicol. 45 (2005) 51-88.

[3] M.J. Snyder, D.R. Maddison, DNA Cell. Biol. 16 (1997) 1373-1384.

[4] W.B. Jakoby, Adv. Enzymol. Relat. Areas Mol. Biol. 46 (1978) 383-414

[5] L.F. Chasseaud, Adv. Cancer Res. 29 (1979) 175-274.

[6] B. Mannervik, U.H. Danielson, CRC Crit. Rev. Biochem. 23 (1988) 283-337.

[7] E. Trottier, A. Belzil, C. Stoltz, A. Anderson, Gene 158 (1995) 263-268.

[8] P. Honkakoski, I. Zelko, T. Sueyoshi, M. Negishi, Mol. Cell. Biol. 18 (1998) 5652-5658.

[9] S. Sanders, S.S. Thorgeirsson, Carcinogenesis 20 (1999) 41-49.

[10] S. Kodama, M. Negishi, Drug Metab. Rev. 38 (2006) 75-87.

[11] A.H. Tang, C.-P.D. Tu, J. Biol. Chem. 270 (1995) 13819-13825.

[12] B. Akgül, C.-P.D. Tu, J. Biol. Chem. 279 (2004) 4027-4033.

[13] C.S. Thummel, A.M. Boulet, H.D. Lipshitz, Gene 74 (1988) 445-456.

[14] B. Akgül, C.-P.D. Tu, J. Biol. Chem. 277 (2002) 34700-34707.

[15] H.M. Robertson, C.R. Preston, R.W. Phillis, D.M. Johnson-Schlitz, W.K. Benz, W.R. Engels, Genetics 18 (1988) 461-470.

[16] A. Ullrich, J. Shine, J. Chirgwin, R. Pictet, E. Tischer, W.J. Rutter, H.M. Goodman, Science 196 (1977) 1313-1319.

[17] P. Couttet, M. Fromont-Racine, D. Steel, R. Pictet, T. Grange, Proc. Natl. Acad. Sci. USA 94 (1997) 5628-5633.

[18] C. Bagni, L. Mannucci, C.G. Dotti, F.J. Amaldi, Neuroscience 20 (2000) RC76.

[19] R.D. Klausner, T.A. Rounault, J.B. Harford, Cell 72 (1993) 19-28.

[20] C.Y. Chen, A.B. Shyu, Trends Biochem. Sci. 20 (1995) 465-470.

[21] M.J. Rui“z-Echevarria, S.W. Peltz, Cell 101 (2000) 741-751.

[22] N. Amrani, M.S. Sachs, A. Jacobson, Nat. Rev. Mol. Cell. Biol. 7 (2006) 415-425

[23] P. Anderson, N.J. Kedersha, Cell. Biol. 172 (2006) 803-808.

[24] G. Shaw G, R. Kamen, Cell 46 (1986) 659-667.

[25] C.Y. Chen, A.B. Shyu, Mol. Cell. Biol. 14 (1994) 8471-8482.

[26] J. Ross, Microbiol. Rev. 59 (1995) 423-450.

[27] P.T. Loflin, C.Y. Chen, N. Xu, A.B. Shyu, Methods 17 (1999) 11-20.

[28] A. Jacobson, Mol. Cell 15 (2004) 1-2.

[29] R. Parker, H. Song, Nat. Struct. Mol. Biol. 11 (2004) 121-127.

[30] E. Wahle, U. Kuhn, Prog. Nucl. Acid. Res. 57 (1997) 41-71.

[31] T. Wilson, R. Treisman, Nature 336 (1988) 396-399.

[32] A.B. Shyu, M.F. Wilkinson, Cell 102 (2000) 135-138.

[33] A.M. Zubiaga, J.G. Belasco, M.E. Greenberg, Mol. Cell. Biol. 1 (1995) 2219-2230.

[34] E. Carballo, W.S. Lai, P.J. Blackshear, Science 281 (1998) 1001-1005. 
[35] G. Stoecklin, M. Colombi, I. Raineri, S. Leuenberger, M. Mallaun, M. Schmidlin, B. Gross, M. Lu, T. Kitamura, C. Moroni, EMBO J. 21 (2002) 4709-4718.

[36] X.C. Fan, J.A. Steitz, EMBO J. 17 (1998) 3448-3460.

[37] J. Kim-Ha, J. Kim, Y.J. Kim, Mol. Cell. Biol. 19 (1999) 2505-2514.

[38] B.J. Reinhart, F.J. Slack, M. Basson, A.E. Pasquinelli, J.C. Bettigner, A.E. Rougvie, H.R. Horvitz, G. Ruvkun, Nature 403 (2000) 901-906.
[39] S. Yekta, I.H. Shih, D.P. Bartel, Science 304 (2004) 594-596.

[40] C. Llave, Z. Xie, K.D. Kasschau, J.C. Carrington, Science 297 (2002) 2002-2003.

[41] K.D. Kasschau, Z. Xie, E. Allen, C. Llave, E.J. Chapman, K.A. Krizan, J.C. Carrington, Dev. Cell 4 (2003) 205-217.

[42] Q. Jing, S. Huang, S. Guth, T. Zarubin, A. Motoyama, J. Chen, F. Di Padova, S.C. Lin, H. Gram, J. Han, Cell 120 (2005) 623-634. 